\title{
Interférences
}

Ars scribendi

$5 \mid 2009$

Historia / Persona

\section{Les témoins anonymes de l'histoire dans la Pharsale de Lucain}

Aline Estèves

\section{(2) OpenEdition}

1 Journals

Édition électronique

URL : http://journals.openedition.org/interferences/905

DOI : 10.4000/interferences.905

ISSN : $1777-5485$

Éditeur

HiSoMA - Histoire et sources des Mondes antiques

Édition imprimée

Date de publication : 1 janvier 2009

ISSN : 1777-5485

\section{Référence électronique}

Aline Estèves, "Les témoins anonymes de l'histoire dans la Pharsale de Lucain », Interférences [En ligne], 5 | 2009, mis en ligne le 16 janvier 2018, consulté le 15 septembre 2020. URL : http://

journals.openedition.org/interferences/905; DOI : https://doi.org/10.4000/interferences.905

Ce document a été généré automatiquement le 15 septembre 2020.

Tous droits réservés 


\title{
Les témoins anonymes de l'histoire dans la Pharsale de Lucain
}

\author{
Aline Estèves
}

1 L'intervention de personnages anonymes relève dans l'épopée d'une tradition admise depuis Homère. L'Iliade comporte en effet des prises de parole qui ne sont le fait ni du poète narrateur, ni des héros : elles sont attribuées à un personnage qu'aucun indice patronymique ou généalogique ne permet d'identifier. Désigné par l'indéfini tis et introduit par quelques formules stéréotypées, il s'agit selon les uns d'une seule personne, selon les autres d'un personnage collectif. Son rôle consiste à commenter brièvement et sporadiquement l'action, en tenant des propos de ton varié, qui englobent sarcasmes et jeux de mots, proverbes ou prières ${ }^{1}$.

2 Si l'on ne peut guère se prononcer, dans l'état actuel des textes, sur la pérennité d'un tel procédé chez les poètes épiques latins jusqu'à Virgile, nous pouvons à tout le moins en relever l'héritage dans l'Énéide: en dehors des interventions malfaisantes de Fama ${ }^{2}$, le poète augustéen évoque par endroits la réaction de personnages dont le nom n'est pas cité, mais dont le propos constitue, comme dans les épopées homériques, un commentaire de l'action ${ }^{3}$.

3 Virgile travaille cependant le motif de manière plus stylisée que le poète grec. En effet, les interventions anonymes ne sont jamais dans l'Énéide le fait d'un personnage isolé, mais toujours d'un groupe de personnes appartenant à une catégorie sociale inférieure à celle des héros - piétaille, femmes, paysans, jeunes gens, etc. Leurs propos, généralement brefs, sont rapportés au style indirect et n'offrent pas une aussi grande variété de ton que chez Homère : ils déplorent le plus souvent les décisions ou l'attitude des héros, mais ils n'ont en aucun cas le pouvoir de les rectifier, si bien que leur rôle semble plus secondaire encore dans l'Énéide que dans l'Iliade. Ils incarnent, en somme, une forme de sagesse populaire qui humanise l'action épique, en lui donnant un éclairage plus accessible à l'homme du commun, et qui dans le même temps sacralise la geste héroïque, en démontrant par contraste combien les réactions spontanées des êtres qui en sont socialement exclus peuvent se révéler aveugles à la grandeur providentielle du destin. 
Par comparaison, fort apparaît le contraste travaillé par Lucain dans la Pharsale ${ }^{4}$ : les personnages anonymes y sont fréquemment sollicités, et leurs paroles ou leurs actions longuement évoquées, qu'il s'agisse de soldats sans nom ni généalogie, ni même patrie ou parti désignés, ou de simples civils non identifiés, exclus des affrontements, mais qu'agitent diverses émotions inspirées par les atrocités d'une guerre qu'ils s'apprêtent à subir ${ }^{5}$.

5 De nombreux critiques se sont penchés sur le cas des soldats anonymes, très représenté dans la Pharsale: ils ont démontré que cet anonymat participait de la déstructuration du schéma héroïque traditionnel de l'épopée, à laquelle recourt le poète néronien pour mieux dénoncer l'horreur insupportable, sur le plan idéologique comme sur le plan humain, des guerres civiles qui ont fait achopper le cours providentiel de l'histoire romaine ${ }^{6}$.

6 Mais Lucain confie également un rôle intéressant à un petit nombre de gens du commun issus de la société civile, dont le cas a été moins étudié ${ }^{7}$ : tout en les maintenant dans l'anonymat et en les excluant de toute participation active à la guerre, il leur attribue une voix singulière, en faisant prononcer à ces personnages a priori inconsistants de véritables discours, rapportés au style direct, dans lesquels ils évoquent, non sans émettre un point de vue personnel, les res gestae de Rome ${ }^{8}$. La majorité des discours concernés par ce cas de figure ${ }^{9}$ sont d'une longueur conséquente - signe que Lucain leur confère une place qui n'a rien de secondaire ${ }^{10}$. Simples témoins anonymes des événements, les individus qui les prononcent sont ainsi érigés en juges de l'histoire romaine dont ils restituent des pans sélectifs.

7 Le lecteur se trouve invité de la sorte à interroger la nature et la portée d'un discours comprenant la mention de res gestae, lorsqu'il est énoncé par d'humbles civils qui, sur le plan narratif, se situent, même temporairement, en marge de l'action militaire dans la Pharsale, et auxquels, sur le plan social, l'écriture historique ne saurait traditionnellement être dévolue à Rome ${ }^{11}$. Quelle mémoire du passé ces personnages conservent-ils? Analysent-ils les événements autrement que les agents de la guerre? Leurs propos relèvent-ils d'un simple témoignage, voire d'une interprétation purement personnels, ou donnent-ils à entendre une voix jusqu'ici inaudible et une vision singulière de la guerre civile ${ }^{12}$ ? L'étude des discours concernés dans l'ordre chronologique où ils se présentent permet d'envisager la progression des propos que Lucain attribue aux civils anonymes, au fur et à mesure qu'évolue la guerre civile.

\section{Les habitants d'Ariminum ${ }^{13}(\mathrm{I}, 244-261)$}

8 Le premier discours que Lucain attribue à des civils anonymes évoquant les res gestae de Rome est tenu, dès le premier tiers du livre I, par les habitants d'Ariminum; ce " peuple» (populus, v. 239) laisse entendre la voix des « jeunes gens » en âge de partir à la guerre (iuuentus, v. 239). À la vue des troupes de César qui occupent leur forum, sitôt le Rubicon franchi, ils entonnent collectivement ${ }^{14}$, comme en rend compte le pluriel de plusieurs verbes renvoyant à l'acte d'énonciation (uoluunt, v. $246:$ «ils exhalent [des plaintes]»; nos ... sumus, v. 248-249: «nous sommes»; nos ... uidimus, v. 254: « nous avons vu»), un discours de déploration (questus, v. 247 : "plaintes»; dolori, v. 258 : «douleur »), que marque dès l'exorde l'expression d'une tristesse funèbre ( 0 male, v. 248 : « pour notre malheur »; O tristi loco, v. 249 : « pour cette funeste situation »). 
9 Leur propos est assez clair: ils expriment le sentiment que leur cité est maudite (damnata, v. 249 : «condamnée »), sa position géographique, aux portes du Latium, condamnant les habitants à subir les premiers assauts de tout ennemi parti attaquer Rome, quand le reste du monde vit dans la paix et la tranquillité - argument développé au moyen d'un parallélisme de construction, v. 249-251 ${ }^{15}$, qui rend le contraste d'autant plus frappant. Ils étayent ces considérations de quelques exemples empruntés au passé de Rome, soulignant à plusieurs reprises, par la répétition de la famille lexicale et le positionnement des termes en fin de vers (furentum, v. 250: «des furieux»; furentem, v. 254 : «en furie »; furoris, v. 255: "de la fureur»), la folie forcenée qui caractérisait ces ennemis de la Ville : sont cités successivement, sans grand respect de l'ordre chronologique, les Sénons, les Cimbres, les Libyens et les Teutons (v. 254-256). Les diverses guerres auxquelles il est fait allusion remontent pour les unes plusieurs siècles en arrière, pour d'autres au passé plus récent de Rome: les Sénons sont les Gaulois menés par Brennus qui s'emparèrent de Rome et pillèrent la cité au $\mathrm{IV}^{\mathrm{e}}$ siècle av. J.-C. ; les Cimbres et les Teutons avaient été défaits au début du I $^{\text {er }}$ siècle av. J.-C. par Marius, après avoir infligé de lourdes pertes aux troupes romaines ; quant au Mars de Libye, il s'agit d'Hannibal ou peut-être de Jugurtha, dont on connaît les exploits respectifs ${ }^{16}$. Autant de guerres qui, en mettant Rome en danger, ont vu s'illustrer contre des ennemis barbares, au cours de bella externa couronnés de succès, des généraux romains héroïques - Camille, Marius, Scipion l'Africain et Sylla -, mais tous soupçonnés de se comporter par la suite en imperatores agissant regio more ${ }^{17}$. Loin de procéder à une reconstitution historique des res gestae de Rome, les habitants d'Ariminum se contentent donc d'allusions fragmentaires témoignant d'une mémoire parcellaire, assez approximatives quant à leur ordonnance chronologique, et dont le rappel a vocation plus pathétique qu'informative.

10 Or les événements qui suscitent ce souvenir révèlent une approche de l'histoire contemporaine lourde d'enseignements : la vue de César parmi les troupes occupant leur forum est à l'origine de leurs plaintes, comme le souligne le narrateur v. 244-247 ${ }^{18}$, avant de donner la parole aux civils d'Ariminum. Lucain spécifie pourtant, non sans intention sarcastique ${ }^{19}$, que César apparaît bien visible au milieu de troupes dont les enseignes sont reconnues ${ }^{20}$. Mais les habitants d'Ariminum ne désignent pas l'armée qui les occupe du terme de Romanus, comme l'avait fait le narrateur ; les premiers mots qu'ils prononcent relient le malheur propre à leur cité à la proximité de leurs voisins les Gaulois ${ }^{21}$ - assimilant ainsi l'armée de César aux armées barbares contre lesquelles se sont illustrés par le passé des généraux romains ${ }^{22}$. Ils proposent de la sorte une interprétation particulière des événements : ils font de César marchant contre Rome une sorte de barbare dominé, comme tous les ennemis de Rome, par le furor ${ }^{23}$; son furor excède cependant celui des barbares, puisque César ramène à Rome des peuples que les généraux antérieurs avaient chassés, manifestant ainsi une déviance contre nature, qui heurte le sens de l'histoire ${ }^{24}$.

11 Dans le temps du récit, les propos des Ariminenses n'ont aucune incidence notable, pour une raison aussi particulière que surprenante: bien qu'il s'agisse d'une plainte collective, c'est-à-dire exprimée et partagée par l'ensemble des habitants, Lucain insiste sur le fait qu'elle n'a pas été prononcée à voix haute ${ }^{25}$, en utilisant un abondant champ lexical du silence et sans craindre les tautologies (tacito in pectore, mutos questus, v. 247 : «dans leur poitrine silencieuse ", "plaintes muettes »; gemitu latenti, v. 257 : " gémissements [poussés] en secret »; non ausus timuisse palam, uox nulla dolori / credita, 
v. 258 : «personne n'osa craindre tout haut », «nul ne confia à la parole sa douleur »; rura silent, tacet sine murmure pontus, v. 260 : «les campagnes sont silencieuses », «la mer est muette et sans mugissement »). Ces personnages sans nom sont en effet surpris au saut du lit par l'occupation de leur forum, à laquelle leur armurerie obsolète ne leur aurait pas permis de résister. Témoins démunis des événements historiques, leur rôle se limite à une attitude totalement passive: simples spectateurs assistant aux faits (conspectus, v. 245: "vu»; tueri, v. 253 : «regarder/garder»), ils émettent à mots couverts une opinion critique qu'ils gardent pour eux. Néanmoins, leur interprétation n'est pas erronée; elle est même cautionnée par le narrateur qui, dans l'exposé des causes de la guerre qui précède, au livre I, a fait du furor le symbole dramatique du dérèglement forcené qui s'empara des généraux et de leurs armées à l'occasion des guerres civiles ${ }^{26}$.

Quelle signification accorder, dès lors, à la situation d'interlocution paradoxale sur laquelle joue Lucain, en livrant au lecteur un discours qui juge de manière assez lucide les res gestae contemporaines à la lumière des res gestae anciennes, mais en niant dans le même temps l'émission explicite de ces propos? Le poète entend sans doute illustrer le régime de terreur qu'impose le déclenchement des hostilités de la part de César ${ }^{27}$. L'intention polémique de Lucain se double d'une intention symbolique : si les habitants d'Ariminum gardent leurs plaintes pour eux, peut-être est-ce parce que personne n'est capable d'entendre, ni de comprendre leur propos. De fait, ils ne visent d'autres destinataires qu'eux-mêmes ou la Fortune, qui ne les a jamais favorisés ${ }^{28}$; leur constat désolé se double d'une récrimination, portant sur la malédiction qui pèse sur leurs murailles, male condita et damnata : les termes ne sont pas sans évoquer, en miroir, les murailles de Rome. Si les murs d'Ariminum sont source de malheurs parce qu'exposés à la moindre offensive barbare, qu'en sera-t-il des murailles de Rome, qu'entache le sang fraternel? Aux premières loges des offensives que comprend le bellum ciuile, les Ariminenses, plus que la voix seule de leur peuple, font sans doute entendre, encore en sourdine, la voix du peuple romain - une voix de souffrance, dont le témoignage, s'il ne repose pas sur une reconstruction ordonnée des événements, livre cependant une vision pathétique et une interprétation lucide de l'histoire romaine ${ }^{29}$.

\section{La matrone romaine $(I, 673-695)$}

13 La fin du livre I complète l'éclairage qu'offrent les propos des habitants d'Ariminum, concernant le rapport qu'entretiennent res gestae, historia et persona du point de vue des victimes civiles : une matrone, saisie de délire divinatoire, parcourt la ville de Rome telle une bacchante; sous l'influence de Phébus, elle évoque pêle-mêle différents lieux et événements qui vont ponctuer le déroulement de la guerre civile ${ }^{30}$. Il ne s'agit donc plus d'aborder le passé ou le présent historiques de Rome, mais le futur. Ce futur, au moment où sont rapportés les propos de la matrone, a déjà pris des couleurs sombres : la terreur dans Rome est telle que le peuple s'enfuit en manifestant un comportement qui prend l'exact contre-pied d'Énée au départ de Troie ${ }^{31}$; de nombreux prodiges ont annoncé les malheurs imminents. Le délire prophétique de la matrone anonyme est présenté par Lucain comme un phénomène couronnant en amplification (maiora, v. 674 : «plus graves ») ces funestes présages - et c'est du reste sur ses sombres paroles que se clôt le livre I ${ }^{32}$. 
La matrone, parmi les visions que lui envoie la divinité, peut en identifier certaines (uideo, v. 679: «je vois»; agnosco, v. 686: «je reconnais »; uidi iam, v. 694: «j'ai déjà $\mathrm{vu} »$ ), tandis que d'autres restent pour elle énigmatiques (quis furor, doce, v.681: "qu'est-ce que cette fureur», "dis-le moi »). Les images que lui transmet Phébus peuvent en effet lui paraître obscures tant que les événements ne les auront pas éclairées, d'autant que sa prophétie reconstitue les principaux événements de la guerre civile à partir des lieux qui en seront le théâtre, si bien que la chronologie n'est pas toujours respectée : partant de la Grèce (batailles de Philippes et Pharsale), passant en Égypte (occupation du palais de Cléopâtre, décapitation de Pompée), sur les côtes d'Afrique du Nord (Utique, Thapsus), puis repassant en Europe, sur la côte méditerranéenne (siège de Marseille et Munda), les visions se terminent en se recentrant sur l'évocation de Rome ${ }^{33}$.

15 À bien y regarder, il s'agit d'un parcours circulaire, ce qui n'est pas dénué d'intérêt sur le plan symbolique: les guerres civiles partent de Rome pour y revenir; elles commencent avec deux armées romaines qui s'entretuent, culminent avec la décapitation de Pompée, événement fortement mis en valeur par le relief plastique conféré aux vers 685-686 ${ }^{34}$, et recommencent avec la conjuration et le meurtre de César, auxquels il est fait allusion en fin de parcours. Est ainsi mise en exergue une conception cyclique de l'histoire de Rome, que les historiens romains développaient depuis l'éclosion des guerres civiles ${ }^{35}$, et dont Lucain se fait aussi l'écho dans la Pharsale, notamment en rattachant la guerre civile de 49 au meurtre fratricide inaugurant la cité romaine ${ }^{36}$.

16 Pour le lecteur, il est évident que sont recensées de la sorte les étapes principales de la guerre à venir et que Lucain esquisse son "programme», quant aux événements historiques que son sujet lui impose d'aborder. Pourquoi, cependant, annoncer ce programme par l'intermédiaire d'un discours prophétique prêté à une matrone anonyme? Pour le comprendre, il convient d'en revenir à la situation d'interlocution. Les propos de la matrone ont un destinataire, la plèbe, sur laquelle ses paroles ont un effet singulier (maiora premunt, v. 674 : «de plus graves [présages] l'accablent »), alors même que la plupart des informations que recèle le discours devraient être énigmatiques pour les protagonistes de l'action. Il est vrai que les mentions géographiques sont entrecoupées d'hypotyposes très succinctes, mettant en images des scènes de violence parfaitement explicites, le combat de deux armées romaines et le tronc décapité d'un homme assez important pour que la matrone le reconnaisse ${ }^{37}$; en fin de passage, c'est la réduplication du motif de l'affrontement duel (consurgunt partes iterum, rursus eo, v. 692-693 : « les partis se lèvent de nouveau ", « je vais à nouveau ») et le jugement condamnant la nature des affrontements ainsi menés (impia bella, v. 691 : « des guerres impies »), qui prend le pas sur les images d'agressivité, mais l'objectif est le même: transmettre une vision pathétique et dramatique de la guerre civile, sous forme de flashes successifs dont l'ordre chronologique importe moins que la réduplication dans l'horreur - discours impressif propre à émouvoir la foule, et par conséquent à la convaincre que ces visions sont à tout le moins vraisemblables, sinon véridiques. En ce sens, les derniers mots du personnage ${ }^{38}$ doivent se comprendre non comme l'expression d'une curiosité, mais comme la formulation d'une prière, visant à ajourner le renouvellement des horreurs cycliques, pour faire place au renouveau.

17 Certes, la matrone anonyme ne résume pas ce faisant l'histoire factuelle : elle prononce une prophétie, forme de discours qui habituellement s'oppose au discours historique; 
elle ne s'appuie sur aucune source, et ses propos ne sont qu'une description succincte des visions qui lui sont imposées ; inspirée par Apollon, telle Cassandre annonçant les malheurs de Troie, elle évoque, sans explication aucune, manifestant même son incompréhension, les multiples et atroces épreuves que l'avenir réserve aux Romains. Mais à la différence de Cassandre, que personne n'avait crue, la matrone accentue l'angoisse du peuple, et le lecteur de Lucain ne sait que trop bien que ses visions reposent sur un fond de véracité - si bien que la prophétie a valeur d'histoire : même incompréhensibles pour les protagonistes de l'action, les malheurs à venir apparaissent non seulement inéluctables, mais sont pour ainsi dire avérés par anticipation ${ }^{39}$. Le fait que Lucain attribue ces propos à une femme est à cet égard signifiant: son incompréhension devant l'affrontement de deux troupes romaines ne vient-elle pas du fait que les femmes, dans l'histoire légendaire de Rome, ont été à plusieurs reprises pour le peuple romain à l'origine de tensions, mais aussi de réconciliations ${ }^{40}$ ? Lui faire prophétiser les malheurs de la guerre civile, n'est-ce pas une manière de dire que le peuple, et les femmes en particulier, entrevoient la tragédie à venir avec une lucidité que leur furor empêche les soldats et leurs chefs d'envisager?

\section{Les Romains partant au combat (II, 43-64)}

18 La signification idéologique et morale que Lucain assigne aux deux premiers discours, dans lesquels le peuple anonyme se réapproprie l'histoire pour y déceler des enseignements qui échappent aux dirigeants et aux soldats, est paradoxalement confirmée par le discours suivant ${ }^{41}$. Lucain évoque au début du livre II le départ à la guerre, vers des camps qu'identifie leur seul antagonisme, des Romains en âge de combattre : envisageant l'avenir avec une triste lucidité ${ }^{42}$, cette piétaille sans nom exprime à son tour des querellas collectives (v. 44 et v. 63 : « reproches", "plaintes »), sans distinction de camp; elle se désole de participer d'un bellum ciuile, au point de regretter les ennemis et les batailles qui faillirent décimer le peuple romain au cours des bella externa du passé ${ }^{43}$, et souhaite dans le même temps que les dieux se contentent de décimer les chefs pour décider de l'issue de la guerre, sans y mêler le peuple romain ${ }^{44}$.

19 Ces hommes font référence au passé de Rome en proposant un nouvel étagement des valeurs: alors même, par exemple, que les défaites de la Trébie et de Cannes constituent un traumatisme effroyable, pour les Romains de la République comme pour les auteurs qui avaient consacré un ouvrage historique à ces événements ${ }^{45}$, elles sont présentées par ces civils qui n'ont pas encore revêtu les armes comme une forme de combat enviable, dans la mesure où les guerres civiles produiront un résultat bien pire ${ }^{46}$; de la sorte, ils proposent à la lumière du présent une nouvelle lecture du passé. D'autre part, leur souhait de voir les ennemis les plus belliqueux (peuples vivant aux confins de l'Empire, Suèves, Daces, Gètes) se coaliser contre Rome prouve qu'ils ont intégré que la guerre civile, pour être l'affaire de deux partis et de deux chefs, n'allait pas moins ravager l'ensemble $d u$ monde romain - issue qu'ils incriminent par anticipation, usant à deux reprises de l'expression bellum ciuile (v. 53 et v. 62) et de termes d'accusation visant à dénoncer aussi bien les forfaits à venir (nouorum ... scelerum, v. 60-61: «de crimes inouïs») que Mars, ancêtre divin auquel une épithète dépréciative ôte tout caractère protecteur, ne retenant de lui que sa cruauté (saeue parens, v. 59 : «Père cruel »). 
20 Les hommes qui profèrent de telles plaintes n'ont pas encore combattu; ils n'ont donc pour ainsi dire pas encore été contaminés par le furor de la guerre civile. Certes, leurs propos trahissent qu'ils sont déjà victimes d'un phénomène propre aux guerres civiles, dont avaient parlé Thucydide et Platon - le renversement des valeurs habituellement admises ${ }^{47}$. Mais le raisonnement auquel ils aboutissent (les défaites passées constituent un sort enviable au regard des temps présents) est présenté par Lucain, en ouverture et en fin de discours, comme un mode de pensée procédant d'une appréciation correcte des événements (iustas ... querellas, v. 44 : «justes reproches ») et d'une forme de pietas (talis pietas peritura querellas / egerit, v. 63-64: «telles sont les plaintes qu'exhale le patriotisme expirant») - valeur en inversion de l'Énéide ${ }^{48}$, puisqu'elle consiste en l'incrimination des dieux, et en un souhait de destruction de la patrie.

21 En somme, par l'intermédiaire de ces hommes anonymes, renâclant à partir au combat, témoins de l'engrenage des guerres civiles avant d'y participer activement, Lucain propose à nouveau une lecture paradoxale du passé de Rome ; le renversement opéré à ce sujet illustre par ricochet l'ampleur des désastres que vont entraîner les guerres civiles. Du reste, ces hommes semblent avoir déjà intégré l'essentiel des propos émis par les civils précédents, de sorte qu'ils les confortent: la guerre civile ne peut être qu'un sujet de douleur et sera d'autant plus destructrice qu'elle s'établit d'emblée à l'échelle de l'univers, tout en dépassant les violences les plus excessives qu'avaient connues jusqu'à présent les Romains. Vision amère d'une histoire dominée, pour ces hommes comme pour Lucain, par le thème de l'écroulement et de la ruine (in se magna ruunt, I, 81 : « les grandeurs s'effondrent sur elles-mêmes »), seules issues possibles.

\section{Le père romain (II, 64-233)}

Lucain développe tout à fait explicitement la cohésion de vue qu'il a progressivement instaurée entre les civils anonymes évoquant l'histoire de Rome et ses propres jugements, à l'occasion du discours suivant, qu'il prête à un homme assez âgé pour avoir connu les proscriptions de Marius et Sylla. La rencontre des deux voix est patente dès les lignes d'introduction : narrateur et personnage interprètent tous deux la guerre civile de 49 comme un renouvellement d'affrontements (iterum bellis ciuilibus, v. 66 : «à nouveau pour les guerres civiles»), dont on peut trouver le modèle dans les proscriptions (exempla, v. 67 : « des précédents »; non alios ... motus, v. $68:$ « c'étaient des mouvements semblables $»)^{49}$.

L'intérêt que présentent les propos du vieillard, eu égard à nos problématiques, tient au fait qu'il se lance dans le récit des proscriptions ${ }^{50}$. Il s'agit en effet du seul personnage anonyme de la Pharsale qui se voit octroyer un tel espace de parole : son récit s'étend sur plus de cent cinquante vers, si bien qu'il s'émancipe de son statut de récit enchâssé - narratio qui, pour comporter nombre de détails d'ordre historique ${ }^{51}$, ne répond cependant pas aux impératifs "scientifiques» de l'historia tels que les développe Cicéron ${ }^{52}$.

24 Le vieillard consacre certes son récit tout entier à l'évocation des proscriptions, dont il retrace les péripéties successives en respectant grosso modo la chronologie ${ }^{53}$ - exil de Marius, rappel de ses exploits sur les Cimbres, retour à Rome et premiers assassinats, mort de Marius et vengeance de Sylla - de sorte que l'unité du sujet apparente le passage au genre de la monographie. Au cours de son récit, le vieil homme évoque du reste la mort des proscrits les plus fameux, livrant ainsi des fragments de biographies ${ }^{54}$ 
- non sans confusion sur leur nom, ni sans erreurs sur l'époque de leur mort pour certains ${ }^{55}$. Chaque fois, le vieil homme signale de quel type d'agression ont été victimes ces hommes (Bébius démembré, Antoine décapité, Crassus disloqué, Scévola égorgé, Marius Gratidianus amputé de tous ses organes) ${ }^{56}$, et développe éventuellement en euidentia ${ }^{57}$ les circonstances morbides dans lesquelles elle s'est effectuée, si bien que les passages s'apparentent, mais de loin seulement, à des notices nécrologiques : les détails macabres leur confèrent une dimension plastique si accentuée que le pathétique l'emporte sur l'informatif, d'autant que la vie de ces personnages n'est pas du tout relatée ; les vignettes en question sont ainsi moins destinées à retracer des événements (docere) qu'à les peindre sous un jour qui suscite haine, dégoût et aversion (mouere) ${ }^{58}$.

$\mathrm{Au}$ demeurant, même si le vieux romain reconstitue la chronologie des proscriptions et cite les morts les plus fameux, il accorde surtout de l'importance aux circonstances pathétiques qui accompagnent ces événements ${ }^{59}$ : la majorité de son développement est ainsi consacrée, pour les proscriptions de Marius, aux meurtres qui touchèrent aussi bien la plèbe que la noblesse (nobilitas cum plebe perit, v. 101 : «la noblesse périt avec la plèbe »), les enfants que les vieillards (nulli sua profuit aetas, v. $104:$ : l'âge ne protège personne »), tandis que les proscriptions de Sylla constituent une surenchère dans l'horreur, puisque les crimes commis le sont entre membres d'une même famille (v. 149-151) ; aux thèmes du charnier et du sang répandu, développés en amplificatio ${ }^{60}$, s'entrelacent trois thématiques dominantes, celles de la tête coupée, du corps défiguré et de l'inversion de l'ordre du monde - les vivants préférant par exemple se cacher parmi les morts et les bêtes sauvages (v. 152-153) -, tous thèmes gratifiés d'un tel développement qu'ils deviennent emblématiques des proscriptions. En insistant sur ces aspects, le vieil homme fait davantage office d'orateur que d'historien: ses propos comportent des éléments relevant des res gestae, mais la reconstitution à laquelle il procède repose moins sur le souci d'instruire en ordonnant et en expliquant que sur la volonté de restituer dans toute son atrocité bouleversante les faits qu'il a vécus et vus de ses propres yeux ${ }^{61}$. Du reste, il parle en qualité de témoin et de victime des proscriptions, insistant sur le fait qu'il a dû lui-même chercher le corps de son frère décapité parmi un monceau de cadavres ${ }^{62}$ - si bien que son discours émane peut-être moins d'une volonté d'émouvoir que de la douleur inspirée par des souvenirs traumatiques ${ }^{63}$.

Il demeure que le vieil homme ne se résout pas à établir la simple chronique des proscriptions. Il propose également au terme de son allocution une interprétation des faits qu'il a relatés, en affirmant que les guerres civiles de 49 , dont les proscriptions furent l'exemplum, dépassent en horreur ces dernières, en raison des motivations qui animent les chefs : Sylla se contentait du pouvoir, Pompée et César, aux dires de cet homme, veulent davantage (neuter ciuilia bella moueret, / contentus quod sylla fuit, v. 231-232: «aucun ne susciterait la guerre civile pour se contenter d'être ce que fut Sylla ») ${ }^{64}$. Autrement dit, la guerre civile de 49 constitue une surenchère dans la surenchère, puisque Sylla avait déjà dépassé Marius en horreur. L'idée est intéressante : elle justifie en quelque sorte les orientations stylistiques du discours du personnage, qui amplifie l'horreur macabre de ses propos au fur et à mesure qu'il progresse dans le récit des proscriptions ; elle explicite aussi les choix stylistiques de Lucain, qui se doit de recourir au tumor pour décrire les excès inégalés, en termes de violence, de la guerre qu'il a choisie pour sujet de son poème. Autrement dit, le témoignage anonyme du 
vieillard n'a rien d'anecdotique: dépassant le simple reportage, sa chronique des guerres civiles a valeur d'analyse historique et de discours idéologique.

Comment concevoir l'histoire de la nation romaine en contexte de guerre civile? Lucain offre une réponse à son lecteur en donnant longuement la parole à des civils exclus des affrontements armés, qui évoquent les res gestae de Rome, mais sont dépouillés de toute individualité: ils n'ont ni patronyme, ni statut social, et ne bénéficient d'aucune précision sur le cours de leur vie qui permette de les individualiser ou de conférer quelque auctoritas à leur propos ${ }^{65}$. S'ils ne se reconnaissent dans aucun parti, ils ne représentent pas non plus une voie alternative à la guerre civile. Ils assistent impuissants à son éclosion, dont le spectacle leur inspire une réflexion d'un nouveau genre concernant les res gestae de Rome : la réduplication de l'affrontement intestin, après les proscriptions de Marius et Sylla, prouve que la mémoire de l'histoire n'a délivré aucun enseignement utile aux hommes qui la font; ceux qui la subissent proposent des événements dont ils sont protagonistes une interprétation qui bouleverse les jugements de valeur traditionnellement admis - les grandes défaites romaines issues des bella externa devenant un objet de louanges, tandis que l'horreur propre aux proscriptions de Marius et Sylla peut être relativisée.

Les propos des Marseillais ${ }^{66}$ tentant de justifier leur pacifisme farouche devant César (III, 306-355), les prédictions de l'augure annonçant la bataille de Pharsale (VII, 192-196), et le discours du satellite de Ptolémée (IX, 1010-1034), qui démontre l'inutilité des bienfaits de Pompée en Égypte, vont dans le même sens, en illustrant la dépossession progressive de son histoire dont est victime le peuple romain, au fur et à mesure que progresse la guerre civile : au livre III, ce ne sont plus des civils romains, mais des Grecs qui revendiquent les valeurs et les faits d'armes relevant traditionnellement de Rome ${ }^{67}$; l'augure du livre VII ne peut que constater, impuissant, l'explosion inéluctable de la bataille emblématique des guerres civiles, à Pharsale ${ }^{68}$ : c'est là la dernière parole de vérité relative, et très brève, que profère un représentant anonyme du peuple romain, dont la voix n'a cessé de s'effilocher ; au livre IX, l'histoire romaine fait l'objet d'une dernière relecture, opérée par le satellite de Ptolémée, qui entérine l'absurdité insupportable du mouvement historique - les bienfaits de Pompée à l'égard de la famille de Ptolémée lui ayant valu d'être ignominieusement assassiné, et les Égyptiens reprenant à leur compte l'action comme l'écriture historique, au point de pouvoir déclarer à César : absenti bellum ciuile peractum est (IX, 1018: « en ton absence, [le roi d'Alexandrie] a terminé pour toi la guerre civile »).

29 Lucain donne ainsi à entendre une lecture de l'histoire issue du prisme collectif, alors même que les guerres civiles avaient surtout fait l'objet de lectures personnelles ${ }^{69}$. La vision et la réception des res gestae changent dès lors de sens: les civils anonymes livrent une vision de l'histoire qui relève d'une intention pathétique, dans un discours $\mathrm{du}$ dolor qui emprunte ses colores à d'autres formes d'expression - tonalité accusatrice issue d'une rhétorique du blâme et tournée vers l'indignatio, tonalité tragique faisant de la guerre civile une sorte de drame familial se jouant à l'échelle de l'univers ${ }^{70}$. Il est significatif, dans cette double perspective, que tout ce que ces personnages anonymes retiennent de l'histoire de Rome, ce soient les grandes défaites et la mort de personnalités, qui émergent en raison non d'un exitu notabili, mais de l'atrocité de leur mort. Tout se passe comme si, parce qu'ils sont dépossédés de la construction de l'histoire et de toute personnalité, les témoins anonymes de la Pharsale pouvaient poser sur leur actualité un regard porteur d'une certaine lux ueritatis ${ }^{71}$, dans la mesure où 
leur discours recroise, sur le plan esthétique comme sur le plan idéologique, celui du narrateur ${ }^{72}$. Néanmoins, leur appréciation reste totalement inutile: sans personne pour les entendre, leur mémoire de l'histoire, du reste fragmentaire, est à l'image des corps fragmentés que suscite la guerre civile - image d'un monde en ruines, défiguré par le furor des appétits personnels ${ }^{73}$.

\section{BIBLIOGRAPHIE}

\section{Textes anciens}

Lucain, La Guerre civile (La Pharsale). I, Livres I-V, éd. A. Bourgery, $2^{e}$ éd. revue et corr., CUF. Série latine, Paris, 1997.

Lucain, La Guerre civile (La Pharsale). II, Livres VI-X, éd. A. Bourgery, M. Ponchont, $6^{\mathrm{e}}$ tirage revu et corrigé, Paris, 1993.

Virgile, Énéide, éd. A. Bellessort, 12e éd., CUF, Paris, 1966, 2 vol. (I, Livres I-VI ; II, Livres VII-XII).

\section{Textes modernes}

AHL F.M. 1976, Lucan: An Introduction, Cornell Studies in Classical Philology 39, Ithaca - Londres. ALBRECHT M. von 1970, «Der Dichter Lucan und die epische Tradition », in M. Durry (éd.), Lucain : sept exposés suivis de discussions, Vandœuvres - Genève du 26 au 31 août 1968, Entretiens sur l'Antiquité classique 15, Genève, p. 267-308.

BOWERSOCK G.W. 1998, «Vita Caesarum: remembering and forgetting the past », in W.W. Ehlers (éd.), La biographie antique, Vandœuvres - Genève, 25-29 août 1997 : huit exposés suivis de discussions, Entretiens sur l'Antiquité classique 44, Genève, p. 193-210.

CARLOZzo G. 1971-1973, « La fama, divinità malefica, in Virgilio ", AAPal 32, 2, p. 145-157.

CASAMENTO A. 2003, « Poeni saturentur sanguine manes (Luc. 1, 39): Lucano, i Mani di Cartagine e i presupposti dell'epos », Pan 21, p. 229-240.

CLÉMENT-TARANTINO S. 2000, « Fama et le poète : pour une poétique de la monstruosité dans l'Énéide ", BAGB 4, p. 309-328.

DANGEL J. 2002, « Fama ("Rumeur") : un emblème virgilien de rhétorique pervertie », in L. Calboli Montefusco (éd.), Papers on Rhetoric, IV, Rome, p. 89-110.

DANGEL J., à paraître, «Lucain et Méduse : les monstres dans l'épopée latine », in H. Karamalengou (éd.), Mélanges en l'honneur de Papadimitriou.

DE JONG I.J.F. 1987, « The voice of anonymity. Tis-speeches in the Iliad », Eranos 85, p. 69-84. 
DELARUE F. 2010, « Les foules de Lucain : émergence du collectif », in O. Devillers, S. Franchet d'Espèrey (éds), Lucain en débat : rhétorique, poétique et histoire, actes du colloque international, Institut Ausonius, Pessac, 12-14 juin 2008, Études 29, Pessac, p. 125-136.

DYER R.R. 1989, « Vergil's Fama. A new interpretation of Aeneid 4.173ff », G\&R 36, p. 28-32.

ERNE M. 1998, «Caractéristiques physiques des femmes selon Tite-Live », EL 1, p. 51-64.

ESPOSITO P. 1981, « Eroi e soldati. Osservazioni sulle battaglie in Virgilio e Lucano », Vichiana 10, p. 62-90.

ESPOSITO P. 1991, « Su una sententia lucanea: Phars. II 14-15 », Vichiana $3^{\mathrm{a}}$ ser. 2, p. 243-252.

ESTÈVES A. 2005, «Le lexique de l'horror dans le B. G. I-VII de César : l'indice d'une anomalie émotionnelle parmi les peurs qu'éprouvent à l'ordinaire les militaires ", in F. Poli,

G. Vottéro (éds), De Cyrène à Catherine : trois mille ans de Libyennes. Études grecques et latines offertes à Catherine Dobias-Lalou, Études anciennes 30, Nancy, p. 333-348.

FRANCHET-D'ESPÈREY S. 2003, «Quis furor, o ciues ? : le furor et la "Furie" comme code poétique de la guerre civile à Rome ", in S. Franchet d'Espèrey et al. (éds), Fondements et crises du pouvoir, É tudes 9, Pessac, p. 429-440.

FRANCHET-D'ESPÈREY S. 2009, « Lucain et le problème de la victoire dans la guerre civile : une théologie de la défaite? », in O. Devillers, J. Meyers (éds), Pouvoirs des hommes, pouvoir des mots, des Gracques à Trajan : hommages au professeur Paul Marius Martin, Bibliothèque d'études classiques 54, Louvain, p. 351-365.

FUGARIU F. 1962, «Le personnage anonyme dans l'épopée homérique », StudClas 4, p. 71-78.

GORMAN V.B. 2000-2001, « Lucan's epic aristeia and the hero of the Bellum Ciuile », CJ 96, 3, p. $263-290$.

GRIMAL P. 1970, «Lucain et l'histoire », in M. Durry (éd.), Lucain : sept exposés suivis de discussions, Vandœuvres - Genève du 26 au 31 août 1968, Entretiens sur l'Antiquité classique 15, Genève, p. 51-117.

HAAG R. 1978, Civil War and the Citizen: Deviations from Some Political Norms in Lucan [Diss. Ohio State University].

JAL P. 1963, La guerre civile à Rome, Publications de la Faculté des lettres et sciences humaines de Paris. Série Recherches 6, Paris.

JOHNER A. 1991, « Rome, la violence et le sacré : les doubles fondateurs », Euphrosyne 19, p. 291-302.

LEDENTU M. 2009, «In arto (...) labor : la parole de l'historien à l'épreuve des guerres civiles et du Principat », in O. Devillers, J. Meyers (éds), Pouvoirs des hommes, pouvoir des mots, des Gracques à Trajan : hommages au professeur Paul Marius Martin, Bibliothèque d'études classiques 54, Louvain, p. 25-38.

LEDENTU M. 2009, «Les Vies de Cornelius Nepos : une nouvelle manière d'écrire l'histoire à Rome? », Interférences 5 [en ligne].

MARTI B.M. 1958, Arnulfi Aurelianensis Glosule super Lucanum, Papers and Monographs of the American Academy in Rome 18, Rome.

MARTI B.M. 1975, « Lucan's narrative techniques », PP 30, p. 74-90.

MASTERS J. 1992, Poetry and Civil War in Lucan's Bellum Ciuile, Cambridge Classical Studies, Cambridge. 
MENDEZ R.D. 1998, « La mujer en Tito Livio », Estudios Clasicos 40, p. 37-46.

MINEO B. 1997, «L'interprétation livienne de l'histoire : le récit des défaites romaines de la Trébie à Cannes », REL 75, p. 113-128.

MINEO B. 2009, « Vies parallèles dans le récit livien : Hannibal et Scipion l'Africain », Interférences 5 [en ligne].

MIURA Y. 1980, «Lucanea. Bellum ciuile and speech of anonymous people », JCS 28, p. 66-76.

MORFORD M.P.O. 1967, The Poet Lucan: Studies in Rhetorical Epic, Oxford.

NARDUCCI E. 1974, « Sconvolgimenti naturali e profezia delle guerre civili. Phars. I, 522-695. Su alcuni problemi di tecnica allusiva nell'epica del primo secolo dell'impero », Maia 26, p. 97-110.

NARDUCCI E. 1979, La provvidenza crudele: Lucano e la distruzione dei miti augustei, Biblioteca di studi antichi 17 , Pise.

NÉRAUDAU J.-P. 1993, « La fama dans la Rome antique », Médiévales 24, p. 27-34.

PAYEN P. 2003, « La guerre des victimes : esprit critique et modèles narratifs dans l'historiographie grecque (ve s. av. J.-C. - II s. ap. J.-C.) ", in G. Lachenaud, D. Longrée (éds), Grecs et Romains aux prises avec l'histoire : représentations, récits et idéologie, I, Actes du colloque de Nantes et Angers, 12-15 septembre 2001, Rennes, p. 203-217.

PICHON R. 1912, Les sources de Lucain, Paris.

RIPOLL F. 2009, « Le problème de la gloire dans le Bellum Ciuile de Lucain », in O. Devillers, J. Meyers (éds), Pouvoirs des hommes, pouvoir des mots, des Gracques à Trajan : hommages au professeur Paul Marius Martin, Bibliothèque d'études classiques 54, Louvain, p. 367-382.

ROWLAND R.J. 1969, « The significance of Massilia in Lucan », Hermes 97, p. 204-208.

SEGAL C. 1971, The Theme of Mutilation of the Corpse in the Iliad, Mnemosyne. Supplementum 17, Leyde.

TUPET A.M. 1981, « La Fama au livre IV de l'Énéide », in R. Chevallier (éd.), Colloque l'épopée grécolatine et ses prolongements européens : Calliope II, Caesarodunum bis 16, Paris, p. 81-91.

WILSON J.R. 1979, « KAI KE TI $\Sigma \Omega \Delta^{\prime}$ EPEEI: An Homeric device in Greek literature », ICS 4, p. 1-15.

\section{NOTES}

1. Fugariu 1962 ; Wilson 1979 ; De Jong 1987 ; Delarue 2010, p. 125.

2. Fama, à bien des égards, représente la voix malfaisante de la foule anonyme : cf. Carlozzo 1971-1973 ; Tupet 1981; Dyer 1989 ; Néraudau 1993, p. 27-29; Clément-Tarantino 2000 ; Dangel 2002.

3. Cf. par ex. Verg., Aen. II, 228-233 (propos anonymes des Troyens commentant la mort de Laocoon) ; II, 238-239 (chants sacrés entonnés par de jeunes gens à l'entrée du cheval de bois dans la cité de Troie) ; V , 4-7 (sombres réflexions des Troyens partis de Carthage à la vue du bûcher de Didon); V, 611-617 (plaintes des Troyennes lasses d'errer sur les mers) ; IX, 450-458 (pleurs et commentaires des Rutules entourant les cadavres de leurs hommes, massacrés par Nisus et Euryale); IX, 498-499 (moment de faiblesse des Troyens, qui expriment leur douleur en entendant les pleurs de la mère d'Euryale) ; X, 262-266 (les Troyens manifestent leur joie et leur élan de bravoure, au retour d'Énée) ; XI, 445-458 (panique des Latins et de leurs alliés à l'annonce d'une offensive d'Énée) ; XII, 1-4 (réprobation hostile des Latins à l'égard de Turnus qui veut 
poursuivre la guerre) ; XII, 216-223 (réprobation des Latins concernant le duel de Turnus et Énée, qu'ils jugent inégal), etc.

4. Sur le traitement de la tradition épique par Lucain, cf. l'article très éclairant d'Albrecht 1970, et en particulier les remarques de P. Grimal dans ce même article, p.307-308, sur le rôle des foules chez Lucain et sa création d'une sorte de "poésie de l'anonyme ».

5. Marti 1975, p. 85-90 ; Miura 1980 (article écrit en japonais, dont nous n'avons pu consulter que le résumé, présenté en anglais) ; Delarue 2010, p. 133.

6. Cf. Esposito 1981 ; Gorman 2000-2001.

7. Cf. Delarue 2010, p. 125-134.

8. Lucain s'inspire peut-être ainsi de l'historiographie grecque, dans laquelle les dérèglements de la guerre sont parfois envisagés à travers la situation des victimes civiles : cf. Payen 2003.

9. Au premier tiers du livre I, les jeunes gens d'Ariminum commentent l'arrivée de César dans leur ville, la comparant au déferlement des troupes barbares qu'ils ont subi par le passé (v. 248-257); à la fin du livre I, une matrone prise de délire divinatoire parcourt Rome et confirme les présages funestes qui ont frappé la cité, ses propos prophétiques annonçant les péripéties essentielles de la guerre civile (v. 679-694) ; au début du livre II, les hommes en âge de combattre, évoquant avec nostalgie les bella externa héroïques du passé de Rome, se lancent dans des récriminations contre le bellum ciuile que leur réserve leur propre époque (v. 45-63), avant que le poète ne donne longuement la parole à un homme assez âgé pour relater la triste histoire des guerres civiles à Rome depuis les proscriptions de Marius et Sylla (v. 68-232) ; au livre III, les Marseillais tentent en vain de fléchir César (v. 306-355) avant de se résoudre à combattre ; au livre VII, un augure annonce que les combats de Pharsale sont imminents (v. 195-196); au livre IX, un satellite de Ptolémée fait valoir devant César son offrande, la tête de Pompée, en évoquant brièvement les bienfaits dont ce dernier avait honoré sa nation par le passé (v. 1014-1032).

10. Delarue 2010, p. 130, estime que ce sont des «personnages collectifs » et « en action », au sens aristotélicien du terme.

11. Sur l'écriture historique comme genre qui est l'apanage de la classe dirigeante sénatoriale à Rome depuis Q. Fabius Pictor, cf. Ledentu 2009, p. 25.

12. Ces questions nous semblent d'autant plus légitimes si l'on tient compte du lien qui existe à Rome entre le discours historiographique et la situation politique. Ledentu 2009, p. 25, signale en effet que «les périodes de crise des institutions et des valeurs [...] trouvent un écho direct et amplifié dans l'historiographie » et s'interroge sur les répercussions qu'a pu avoir la guerre civile «sur l'écriture de l'histoire, sur la manière dont les Romains ont pensé leur rapport avec la mémoire de ces événements ».

13. Sur les sources de Lucain concernant cet épisode, cf. Pichon 1912, p. 98-101. Pour une étude des circonstances dans lesquelles se déroule ce discours, cf. Haag 1978, p. 108-113. Pour Grimal 1970, p. 66-68, les événements d'Ariminum tels qu'ils sont relatés dans la Pharsale reflètent davantage la réalité historique que ne le font les commentaires de César.

14. Delarue 2010, p. 130-132, analyse ce passage, notant que «le collectif ne laisse plus de place à l'individuel ».

15. I, 249-251: pax alta per omnes / et tranquilla quies populos : nos praeda furentum / primaque castra sumus, «une paix profonde et un sommeil sans trouble règne [sic] sur tous les peuples: nous sommes la proie des furieux et leur premier camp ».

16. Concernant toutes ces allusions historiques, cf. le commentaire d'Arnould d'Orléans, in Marti 1958, p. 40.

17. Cf. notamment Mineo 2009. C'est le cas également de Marius, rapproché à plusieurs reprises dans la Pharsale d'Hannibal et de Jugurtha, selon Ahl 1976, p. 103-104: il en est question dans le récit des proscriptions fait par le vieillard anonyme du livre II (cf. infra). 
18. I, 244-247 : ut notae fulsere aquilae Romanaque signa / et celsus medio conspectus in agmine Caesar, / deriguere metu, gelidos pauor occupat artus, / et tacito mutos uoluunt in pectore questus, "quand ils voient briller les aigles bien connues, les enseignes romaines, et César dominant le centre de sa troupe, ils frissonnèrent d'effroi, l'épouvante glace leurs membres, et dans leur poitrine silencieuse s'exhalent ces plaintes muettes ».

19. Sans doute Lucain souhaite-t-il rappeler à son lecteur que César, en relatant dans les Commentarii son offensive finale contre Alésia, avait mentionné l'importance de son apparition en manteau de général au milieu des combats (Caes., B. G. VII, 88, 1 : Eius aduentu ex colore uestitus cognito, quo insigni in proeliis uti consuerat, « on apprit son arrivée à la couleur de son vêtement, signal dont il avait pris l'habitude d'user lors des combats".

20. Cf. Arnould d'Orléans, in Marti 1958, p. 39 : Cesar argumentum a nomine quasi cedens omnia. Celsus quia in quodam colliculo ubi uideretur ab omnibus, uel celsus quia ut dicunt magne stature fuit.

21. I, 248-249: O male uicinis haec moenia condita Gallis, / O tristi damnata loco !, "Ô nos murailles, bâties pour notre malheur avec les Gaulois pour voisins, condamnées pour cette funeste situation! » Concernant les Gaulois et l'excursus que leur consacre Lucain au livre I, dans les vers suivants, alors que César rappelle ses troupes de Gaule après s'être emparé d'Ariminum, cf. Pichon 1912, p. 24-34.

22. La XIII légion gemina avec laquelle il avait franchi le Rubicon comportait en effet des Gaulois recrutés en Gaule cisalpine : cf. Grimal 1970, p. 72, 82-83.

23. Cf. sur ce point Ahl 1976, p. 107 : César devient un alter Hannibal.

24. Delarue 2010, p. 133 : "effroyable paradoxe, ce sont les armes théoriquement amies qui provoquent l'épouvante »; le critique analyse ensuite «l'intertextualité » du passage, qui « vient souligner le monstrum ", concluant qu' " un siècle après les événements, le lecteur romain ne peut que continuer à partager l'horreur d'une telle situation ».

25. Delarue 2010, p.126: « [à Ariminum], le poète pousse plus loin encore le refus de l'individualisation : il crée ce qui, du point de vue de la narratologie, est un véritable monstre, un monologue intérieur [...] collectif au style direct ». Pour Haag 1978, p. 113, « by presenting their secret thoughts, by not identifying any individual, by not portraying even a fictitious character, the poet succeeds in fashioning a completely impersonal - and thereby even more devasting pervasive atmosphere of doom ".

26. I, 8-9: Quis furor, o ciues, quae tanta licentia ferri / gentibus inuisis Latium praebere cruorem !, «D'où vient, citoyens, cette fureur? Où le fer a-t-il pris cette licence d'offrir le sang latin à des peuples qui nous haïssent? » Cf. à ce sujet Franchet d'Espèrey 2003.

27. Il s'agit d'un autre souvenir, peut-être des Commentarii sur la guerre des Gaules, dans lesquels César évoque, au livre I, l'attitude des Gaulois terrorisés par les Germains, au point qu'ils n'osent même prononcer leurs plaintes devant le général romain (B. G. I, 32). Cf. à ce sujet notre article, Estèves 2005. L'inversion à laquelle procède Lucain relève de la charge accusatrice, en allant dans le même sens que les propos qu'il prête aux habitants d'Ariminum. Sur l'attitude des Ariminenses, cf. Arnould d'Orléans, in Marti 1958, p. 40 : Tacito quia non audent aperte conqueri de Cesare.

28. I, 251-253 : Melius, Fortuna, dedisses / orbe sub Eoo sedem gelidaque sub Arcto / errantisque domos, Latii quam claustra tueri, « Tu aurais mieux fait, Fortune, de nous donner une demeure dans le monde oriental ou parmi les glaces arctiques et des maisons errantes, que de nous faire surveiller les verrous du Latium ».

29. Delarue 2010, p. 133 : «pour Lucain, il en va de même pour les cités et les foules : de même que les Marseillais, les Mytiléniens ou les soldats qui, en Espagne, fraternisent, les Ariminenses sont des personnages "de qualité", exemples de la conduite qui devrait être celle de tous les citoyens".

30. Sur ce passage et la confusion Philippes-Pharsale, cf. Ahl 1976, p. 314-315.

31. I, 503-509 : sic Vrbe relicta / in bellum fugitur. Nullum iam languidus aeuo / eualuit reuocare parens coniunxue maritum / fletibus, aut patrii, dubiae dum uota salutis / conciperent, tenuere lares; nec limine 
quisquam / haesit et extremo tunc forsitan urbis amatae / plenus abit uisu ; ruit inreuocabile uolgus, « de même, laissant la Ville, on fuit vers la guerre. Un père accablé d'ans n'a plus été capable de ramener un fils, ni l'épouse en pleurs un mari, les lares paternels n'ont pas assez retenu, pour formuler des vœux d'un salut incertain, personne ne s'arrêta sur le seuil, personne à l'heure du départ ne se rassasia de la contemplation - la dernière peut-être - d'une ville aimée ; la masse se rue sans retour $»$.

32. Cf. sur ce point Pichon 1912, p. 186-188 : le livre I comporte trois prédictions, fournies par Arruns, Nigidius Figulus et la matrone, qui gagnent à chaque fois en précision et deviennent de plus en plus dramatiques. Cf. également Morford 1967, p. 64-65, sur la construction du livre I et l'intensité dramatique, sur le plan stylistique, de ce passage.

33. Pour l'explicitation des lieux et événements concernés, cf. Arnould d'Orléans, in Marti 1958, p. 86-88.

34. I, 685-686: hunc ego, fluminea deformis truncus harena / qui iacet, agnosco, « celui qui gît, tronc informe, sur les sables du fleuve, je le reconnais ».

35. Cf. Dangel (à paraître) : "alors que Virgile réfléchissait sur les fondements historiques de Pharsale et Philippes fusionnant dans la Thessalie hémonienne, Lucain insiste sur une récidive antérieure à l'histoire, si bien qu'elle paraît inexorable : les actes fondateurs sont contenus dans le mythe ». Cf. également Jal 1963, p. 407 ; Johner 1991.

36. I, 95 : fraterno primi maduerunt sanguine muri, «nos premières murailles ont été tachées du sang d'un frère $"$.

37. Cf. n. 26 et 34 .

38. I, 693-694: Noua da mihi cernere litora ponti / telluremque nouam: uidi iam, Phoebe, Philippos, « fais-moi voir de nouveaux rivages et une nouvelle terre, Phébus : j'ai déjà vu Philippes ».

39. Sur la relation mythe-histoire qui sous-tend ce passage et a fait sa fortune, notamment chez Stace, cf. Narducci 1979, p. 153, 161.

40. Cf. à ce sujet Mendez 1998 ; Erne 1998.

41. Ahl 1976, p. 234-235.

42. Dès le début du livre II, Lucain a lié la lucidité des hommes vis-à-vis des malheurs qui les attendent à un état de désespoir et de tristesse funèbre (II, 14-15: sit caeca futuri / mens hominum fati; liceat sperare timenti). Pour une étude de ce lieu commun tel que le réinvestit Lucain, cf. Esposito 1991, p. 247-252.

43. Cf. Arnould d'Orléans, in Marti 1958, p. 94 : Non pacem petimus quasi nos uolumus bellum cum probi simus sed tale, id est illicitum et ciuile, abhorremus.

44. Peut-être faut-il voir dans ce souhait irréalisable le souvenir de la solution virgilienne, qui dans l'Énéide met fin à la guerre fratricide du Latium avec le duel de Turnus et d'Énée.

45. Sur la bataille de Cannes chez Tite-Live, dont le récit est structuré par la problématique discorde/concorde, cf. Mineo 1997.

46. II, 60-63: Tantone nouorum / prouentu scelerum quaerunt uter imperet Vrbi ? / Vix tanti fuerat ciuilia bella mouere / ut neuter, «Faut-il une telle abondance de crimes inouïs pour chercher lequel commandera Rome? À peine eût-il fallu susciter des guerres civiles pour que ce fût ni l'un ni l'autre".

47. Platon, Rép. III, 560d3-8; Thucydide, III, 82, 4.

48. Du reste, Lucain construit dans la Pharsale un discours historique et idéologique, concernant les guerres puniques, qui prend le contre-pied de l'Énéide : cf. Casamento 2003, p. 232-236.

49. Grimal 1970, p. 89, insiste sur le fait que cela n'a rien de rhétorique : le rapprochement s'était instauré à Rome, parmi la population, et correspond donc à une forme de réalisme psychologique voulu par Lucain.

50. Je remercie M. Ledentu de m'avoir signalé que ce personnage qui narre les proscriptions de Marius et Sylla et s'érige ainsi en historien d'une partie des guerres civiles renvoie peut-être, dans l'esprit de Lucain, à Sénèque le Rhéteur, un des rares historiens de Rome qui se soient 
exprimés sur le sujet; cf. Ledentu 2009, p. 33-34 : « ... les historiens, après la mort de César et l'avènement au pouvoir d'Octave, ont dû se poser la question du statut de la guerre civile dans la mémoire historiographique de l'Vrbs. [...] Face au Bellum Ciuile de César et avant la réponse que Lucain, poète-historien, y apportera à l'époque néronienne, rares ont été ceux qui, parmi les nombreux auteurs à avoir écrit l'histoire, ont osé narrer l'épisode des guerres civiles. [...] Trois écrivains nous sont connus pour avoir eu le courage et trouvé l'opportunité de traiter ce sujet : Asinius Pollion, Tite-Live, Sénèque le Rhéteur ». Ce dernier aurait été publié au début du règne de Caligula : cf. ibid., p. 37.

51. Selon Pichon 1912, p. 14-15, la source principale de ce passage, sur le fond, est Tite-Live, et «le propos de Lucain s'accorde aussi bien avec cet historien qu'avec ceux qui se sont inspirés de lui, Velleius, Florus, Appien, Dion, Plutarque, etc.», même si «l'accord n'est pas complet » et si " on peut relever des divergences ", « légères et aisément explicables ».

52. Cicéron, De oratore II, 51-64.

53. Sur les sources de ce passage, cf. Pichon 1912, p. 13-16.

54. Sur le genre biographique à Rome, cf. Bowersock 1998, ainsi que l'article de Ledentu 2009.

55. Cf. sur ce point les précisions de Pichon 1912, p. 15-16 (confusions sur Baebius, Scaevola, Marius).

56. Il s'agit d'autant d'atteintes qui signalent un surcroît de violence dans l'épopée ; cf. à ce sujet Segal 1971.

57. Sur l'euidentia, qui consiste à évoquer une scène de manière à donner l'illusion à l'auditeur qu'elle se déroule sous ses yeux, cf. Quint., I.O. VI, 1, 15-17 ; VIII, 3, 61-69 ; IX, 2, 40.

58. Les lois du genre historique veulent que l'auteur écrive sine ira et studio (préfaces de Salluste, Tite-Live, Tacite).

59. Pour Pichon 1912, p. 14, on décèle là une écriture dramatique de l'histoire qui correspond peu ou prou à la manière historique de Tite-Live.

60. Cf. le Tibre dont le flot déborde en raison du monceau de cadavres qui y a été jeté, v. 209-220.

61. Le discours du vieillard repose en effet sur différents lieux de l'amplification décrits dans les traités de rhétorique : cf. par ex. Cic., Invent. I, 104 ; Quint., I.O. VI, 2, 31-32.

62. II, 169-173: Meque ipsum memini, caesi deformia fratris / ora rogo cupidum uetitisque inponere flammis, / omnia Sullanae lustrasse cadauera pacis / perque omnis truncos, cum qua ceruice recisum / conueniat, quaesisse, caput, " Moi-même, il m'en souvient, soucieux de placer sur le bûcher et les flammes interdites le visage défiguré d'un frère massacré, je parcourus tous les cadavres de la paix de Sylla et cherchai parmi tous les troncs à quel cou convenait la tête coupée ».

63. Cf. Arnould d'Orléans, in Marti 1958, p. 106 : meque ipsum memini probat hoc auctoritate sua, quasi diceret : experto credite, nam memini me lustrasse cadauera.

64. Cf. Arnould d'Orléans, in Marti 1958, p. 111 : Contentus quo Silla fuit id est potencia penitus tollendi partes inuisas.

65. L'auctoritas de l'auteur est essentielle pour imposer son discours historique. Ledentu 2009, p. 35, analyse par ex. le cas très particulier d'Asinius Pollion, qui fut toute sa vie opposé à OctaveAuguste, mais qui put écrire sur les guerres civiles : « si Pollion pouvait s'autoriser à écrire sur un tel sujet, c'est en raison de son auctoritas d'homme politique, de la relative immunité dont il jouissait auprès d'Auguste et de son statut de témoin des événements, présent sur le théâtre des combats, qui lui permettait de faire valoir la véracité de son récit ».

66. Cf. Ripoll 2009, p. 374-375, à propos de cette intervention des Marseillais : «la seule action qualifiée de glorieuse par le narrateur de la guerre civile n'est pas une victoire militaire, mais une action retardatrice : il s'agit du rôle des Marseillais comme force de résistance à César (III, 388-391) [...]. Dans le cas de Marseille comme de Mytilène, la gloire de la collectivité est associée au respect d'une valeur morale romaine particulière (foulée aux pieds par nombre de Romains en ces temps troublés), la fides [...]. Le poète de la Pharsale rééquilibre [ainsi] la balance de la renommée en faveur de l'héroïsme moral aux dépens de la gloire militaire». Cf. également 
Pichon 1912, p.126-128; Delarue 2010, p.125-126; Haag 1978, p.114-123, qui insiste en conclusion sur « the very close connection between Rome and Massilia in Lucan's poetic vision »; Masters 1992, p. 13-19 (sources de Lucain sur l'épisode) et p. 20-25 (comparaison de l'épisode chez César et Lucain).

67. Cf. à ce sujet Rowland 1969.

68. VII, 192-196 : si uera fides memorantibus, augur / colle sedens, Aponus terris ubi fumifer exit / atque Antenorei dispergitur unda Timaui, / "Venit summa dies, geritur res maxima », dixit, / " inpia concurrunt Pompei et Caesaris arma», "S'il faut en croire la tradition, un augure, assis sur la colline euganéenne où l'Aponus sort fumant des terres et où l'onde du Timave d'Aténor se disperse, s'écria : "Il est venu, le jour suprême; la lutte la plus grave est engagée; les armes impies de Pompée et de César s'entrechoquent" ».

69. À ce titre, les propos des civils dans la Pharsale concernant les res gestae de Rome reflètent le malaise idéologique qui s'empara de la nation avec les guerres civiles, où la théologie de la victoire devait être modifiée, en passant d'une victoire nationale à la victoire d'un individu : cf. à ce sujet Franchet d'Espèrey 2009, p. 352-353.

70. Le color tragique est d'autant plus prégnant que les allusions tragiques contenues dans ces discours se doublent de rapprochements interlocutoires : les propos des habitants d'Ariminum s'apparentent ainsi aux déplorations du chœur tragique; le récit des proscriptions rappelle les récits de messagers relatant par le détail et avec force expressivité le nefas tragique ; la prophétie de la matrone s'intègre à une série de prodiges néfastes qui recréent l'atmosphère mythique propre aux drames de Thèbes et Mycènes, selon Narducci 1974.

71. Le rapport de l'historiographie à la vérité est un problème fondamental que posent souvent les historiens à travers leurs préfaces; cf. notamment cette analyse de Ledentu 2009, p. 33 : «La proximité de la formule livienne, non flectere a uero, avec celle de Salluste, neque me mouit a uero, révèle une communauté de réflexion sur le statut de l'engagement historiographique et la valeur de l'écriture de l'histoire comme discours de vérité, dans la Rome traumatisée par l'expérience des guerres civiles et l'affrontement de deux partis, guerre menée par les armes autant que par la propagande idéologique ".

72. Cf. les remarques de Franchet d'Espèrey 2009, p.354-358, sur le discours de Lucain concernant la guerre civile, nefas qui subvertit toutes les valeurs, notamment la uirtus, si bien que le poète en vient à soutenir que «la défaite est une bonne chose et même un titre de gloire équivalent à la victoire [...]. On pourrait parler ici de théologie de la défaite, tant le retournement est spectaculaire ». De même, Ripoll 2009, p. 368-372, fournit cette analyse préliminaire : " Même si l'épopée de Lucain ne comporte que peu d'éléments de réflexion théorique sur le problème de la gloire, on peut y voir en filigrane l'illustration d'un lieu commun de la pensée morale romaine [...] : le fait que la guerre civile est une guerre sans gloire, ou du moins n'est pas pourvoyeuse d'une gloire légitime susceptible d'une célébration épique ». Le seul personnage dont la gloria ne souffre ni bémol ni ambiguïté dans la Pharsale est Caton, lors de sa traversée du désert de Libye et de sa confrontation aux serpents, ibid., p. 368-372, étude lumineuse débouchant sur cette conclusion: " cette véritable aristie de Caton qu'est la traversée du désert libyen au chant IX, figure métaphorique du chemin de la Vertu, est une antithèse à la fois morale et littéraire à l'épopée dévoyée de la guerre civile ».

73. Delarue 2010, p. 134-135, établit le rapport entre le rôle conféré aux collectivités anonymes dans la Pharsale, le prologue de l'œuvre, et l'absence des dieux dans l'épopée. 


\section{RÉSUMÉS}

L'intervention de personnages anonymes relève dans l'épopée d'une tradition admise depuis Homère. L'article les étudie chez Lucain dans l'ordre chronologique où ils se présentent afin d'envisager la progression des propos que Lucain attribue aux civils anonymes, au fur et à mesure qu'évolue la guerre civile. L'étude des propos des civils anonymes livre ainsi une vision de l'histoire qui relève d'une intention pathétique, dans un discours du dolor qui emprunte ses colores à d'autres formes d'expression - tonalité accusatrice issue d'une rhétorique du blâme et tournée vers l'indignatio, tonalité tragique, faisant de la guerre civile une sorte de drame familial se jouant à l'échelle de l'univers.

\section{INDEX}

Mots-clés : colores, euidentia, mouere, personnages littéraires anonymes, res gestae, témoignage

Keywords : colores, euidentia, mouere, anonymous literary character, res gestae, testimony nomsmotscles Appien, César, Cicéron, Dion Cassius, Florus, Homère, Lucain, Plutarque, Quintilien, Sénèque le Rhéteur, Velleius Paterculus, Virgile

Index géographique : Afrique du Nord, Arminium, Égypte, Europe, Marseille, Munda, Pharsale, Philippes, Rome, Troie, Utique, Thapsus

\section{AUTEURS}

\section{ALINE ESTÈVES}

Université Paul-Valéry, Montpellier III 